\title{
Keratan Sulfate Proteoglycan Phosphacan Regulates Mossy Fiber Outgrowth and Regeneration
}

\author{
Christy D. Butler, ${ }^{2}$ Stephanie A. Schnetz, ${ }^{1}$ Eric Y. Yu, ${ }^{1}$ Joseph B. Davis, ${ }^{1}$ Katherine Temple, ${ }^{1}$ Jerry Silver, ${ }^{2}$ and \\ Alfred T. Malouf ${ }^{1,2}$ \\ Departments of ${ }^{1}$ Pediatrics and ${ }^{2}$ Neurosciences, Case Western Reserve University, Cleveland, Ohio 44106
}

\begin{abstract}
We have examined the role of chondroitin sulfate proteoglycans (CSPGs) and keratan sulfate proteoglycans (KSPGs) in directing mossy fiber (MF) outgrowth and regeneration in rat hippocampal slice cultures. MFs normally exhibit a very specific innervation pattern that is restricted to the stratum lucidum (SL). In addition, MFs in hippocampal slice cultures will regenerate this specific innervation pattern after transection. CSPGs are one of the best characterized inhibitory axon guidance molecules in the CNS and are widely expressed in all areas of the hippocampus except SL. KSPGs are also widely expressed in the hippocampus, but their role in axon outgrowth has not been extensively studied in the CNS where phosphacan is the only protein that appears to contain KS-GAGs. Cultured hippocampal slices were treated with either chondroitin $\mathrm{ABC}$ lyase or keratanases to reduce the inhibitory axon guidance properties of CS and KS proteoglycans, respectively. The ability of transected MFs to regenerate their normal innervation pattern after digestion of CS and KS-GAGS sugars with these enzymes was examined. Only keratanase treatment resulted in misrouting of MFs. Identifying the mechanism by which keratanase produced MF misrouting is complicated by the presence of splice variants of the phosphacan gene that include the extracellular form of phosphacan and the transmembrane receptor protein tyrosine phosphatase $\beta / \zeta(\operatorname{RPTP} \beta / \zeta)$. Both forms of phosphacan are made by astrocytes, suggesting that keratanase alters MF outgrowth by modifying astrocyte function.
\end{abstract}

Key words: hippocampus; mossy fibers; regeneration; proteoglycans; axon guidance; phosphacan; neurocan; RPTP $\beta / \zeta$

\section{Introduction}

The mossy fiber (MF) tract is one of the three major axonal pathways in the hippocampus, an area of the brain that plays a central role in learning and memory (Alvarez-Royo et al., 1992; Squire, 1993; Granger et al., 1996). MFs originate from granule cells in the dentate gyrus and synapse on the proximal apical dendrites of CA3 pyramidal neurons within a narrow band called the stratum lucidum (SL). Granule cells are one of two types of CNS neurons that continue to be generated in adult animals (Altman and Das, 1965; Gaarskjaer, 1985; Schlessinger et al., 1993). Thus, MFs from newly generated granule cells must continue to generate their normally restricted synaptic pattern within SL throughout life. The axon guidance mechanisms that enable MFs to continue to innervate their appropriate synaptic targets in vivo are not well understood. However, the same guidance molecules may also help MFs regenerate their normal synapses in cultured hippocampal slices after transection (Zimmer and Gähwiler, 1987; Gaiarsa and Heimrich, 1995; Nguyen et al., 1996; Grimpe et al., 2002).

In spite of their highly restricted synaptic pattern, MFs can generate abnormal synapses in animal seizure models and in patients with temporal lobe epilepsy. In these cases, MFs grow back-

Received June 22, 2003; revised 0ct. 27, 2003; accepted 0ct. 28, 2003.

This work was supported by National Institutes of Health Grant NS 41383 (A.T.M.).

Correspondence should be addressed to Dr. Alfred Malouf, Department of Pediatrics, Case Western Reserve University, 11100 Euclid Avenue, MS 6003, Cleveland, OH 44106. E-mail atm3@po.cwru.edu. DOI:10.1523/JNEUROSCI.3040-03.2004

Copyright $\odot 2004$ Society for Neuroscience $\quad$ 0270-6474/04/240462-12\$15.00/0 wards and synapse on the proximal dendrites of granule cells rather than on CA3 pyramidal neurons. These abnormal synapses form a recurrent excitatory circuit that is thought to enhance the appearance of seizure activity (Sutula et al., 1989; Mello et al., 1993; Mathern et al., 1994; Okazaki et al., 1995). It is important to note that in spite of this backwards MF outgrowth and synaptogenesis, MFs do not exhibit extensive misrouting into other areas of the hippocampus. Thus, MFs appear to be inhibited from making synapses onto the distal dendrites of CA3 pyramidal neurons or onto CA1 pyramidal cells. These observations suggest that inhibitory molecules surround the SL and keep MF outgrowth restricted to the SL in area CA3.

Proteoglycans are good candidates for restricting MF outgrowth. Most proteoglycans are extracellular matrix (ECM) molecules that regulate cell adhesion, migration, and axon outgrowth (Snow et al., 1990; Wight et al., 1992; Margolis and Margolis, 1993; Oohira et al., 1994; Asher et al., 2001). The chondroitin sulfate proteoglycans (CSPGs), such as neurocan and phosphacan, have been reported to inhibit axon outgrowth (Friedlander et al., 1994; Milev et al., 1994). Proteoglycans contain a protein core and are classified by their covalently attached carbohydrates called glycosaminoglycans (GAGs). The inhibitory function of proteoglycans on axon outgrowth has typically been examined by enzymatically removing the GAG sugars from the core protein. The use of chondroitin $\mathrm{ABC}$ lyase and keratanases to enzymatically remove chondroitin and keratan sulfate GAGs, respectively, has provided a powerful method for examining the role of proteoglycans in brain development. For example, enzymatic re- 
moval of chondroitin sulfate GAGs using chondroitin ABC lyase has been shown to disrupt the normal development of retinal ganglion cell axons (Snow et al., 1991; Brittis et al., 1992). CSPGs also appear to be important for regulating fasciculation of axons entering the fimbria during late embryonic and early postnatal hippocampal development (Wilson and Snow, 2000).

In our present study, we have observed that CSPGs are highly expressed in the areas surrounding the SL during the early postnatal period of maximal MF development. This inhibitory CSPG boundary surrounding the MFs in SL was also observed by Kurazono et al. (2001), who suggested that CSPGs play an important role in preventing MFs from sprouting outside of SL after seizure activity. However, presently there is no direct functional evidence for the involvement of CSPGs in directing MF outgrowth during either early or late MF development.

To examine the role of CSPGs in MF outgrowth, we treated cultured hippocampal slices with chondroitin ABC lyase. We also used keratanase to enzymatically remove keratan sulfate GAG sugars from phosphacan, which is the only CNS proteoglycan that contains keratan sulfate GAGs that can be digested by keratanase (Rauch et al., 1991; Maurel et al., 1994). Phosphacan is expressed as three different splice variants in the CNS (Maurel et al., 1994, 1995). There is one extracellular matrix form of phosphacan, whereas the other two forms represent short- and fulllength forms of the transmembrane receptor protein tyrosine phosphatase $\beta / \zeta(\mathrm{RPTP} \beta / \zeta)$. The extracellular domain of fulllength $\mathrm{RPTP} \beta / \zeta$ is identical to the extracellular form of phosphacan.

Our data show that keratanases produced a dramatic change in MF outgrowth and regeneration, whereas chondroitin $\mathrm{ABC}$ lyase appeared to have little effect. These findings provide new information about the role of proteoglycans in the regulation of mammalian CNS axon regeneration.

\section{Materials and Methods}

Cultures. Hippocampal slice cultures were prepared from 4-14 d old Sprague Dawley, rat pups (Zivic Miller, Pittsburgh, PA) (Stoppini et al., 1991). Rats were decapitated, and $400 \mu \mathrm{m}$ transverse hippocampal slices were prepared using a Mcllwain Tissue Chopper. Slices were placed on 25 mm Nunc (Roskilde, Denmark) Anopore Membranes in 6 well tissue culture plates and fed every other day with $1.5 \mathrm{ml}$ growth medium $(50 \%$ MEM, 25\% HBSS, and 25\% horse serum; Invitrogen, Grand Island, NY). The growth medium was supplemented with $4 \mathrm{ml}$ of $50 \%$ sterile dextrose. The cultures were maintained for up to 2 weeks at $36^{\circ} \mathrm{C}$ and $5 \%$ $\mathrm{CO}_{2}$ in a humidified incubator. Hippocampal cultures used in this study had a complete hippocampal anatomy (dentate gyrus, hilus, and CA1 and CA3 pyramidal cell layers). For regeneration experiments, mossy fibers were transected as previously described (Nguyen et al., 1996) and allowed to regenerate for $5 \mathrm{~d}$. Briefly, a cut was made along an axis perpendicular to the pyramidal cell layer and in front of the open end of the granule cell layer using a \#15 scalpel. Dentate explants were prepared by dissecting the dentate from the hippocampus as previously described (Nguyen et al., 1996). Briefly, a cut was made between the dentate and the hippocampus, as described for MF transection experiments. Another cut was then made along the hippocampal fissure, and the dentate was carefully removed from the hippocampus. The dentates were further processed to remove CA3c pyramidal cells and much of the hilus. Thus, dentate explants consisted almost entirely of the dentate granule cell layer and the molecular layers.

Proteoglycan substrate. Before placing the dentate explants on Nunc membranes, the membranes were coated with a $0.01 \%$ solution of polylysine (Sigma, St. Louis, MO) followed by $1 \mathrm{mg} / \mathrm{ml}$ solution of laminin (Sigma). For some experiments, half of the membrane was coated with laminin and the other half with a proteoglycan mixture isolated from embryonic chick brain $(550 \mu \mathrm{g} / \mathrm{ml}$; Chemicon, Temecula, CA). The laminin was always applied first and allowed to dry. The first coat of proteoglycans was applied so that it overlapped the laminin substrate. The proteoglycan mixture was applied to the culture membrane and allowed to dry before a second layer was applied. These layers partially overlapped so that a substrate consisting of $\sim 1 \times$ and $2 \times$ concentrations of proteoglycan was generated. However, the exact concentration of proteoglycan in the substrate is not precisely known. Alexa 594 was added to the proteoglycan or KS mixtures to visualize the location of these substrates relative to axon outgrowth. The membranes were washed with 1 $\mathrm{ml}$ of Neurobasal growth medium and placed in 6 well plates containing $1.5 \mathrm{ml}$ of Neurobasal growth medium (Invitrogen).

The same dissection protocol was used to make dissociated dentate cultures. The dentate tissue fragments were then finely minced using spring scissors. The resulting cell suspension was centrifuged, and the pellet was resuspended by trituration and washed two more times in Neurobasal growth medium before being plated on a glass coverslip coated with polylysine followed by laminin. Each coverslip was placed in one well of a 6 well plate and fed with $1 \mathrm{ml}$ of Neurobasal growth medium.

Immunohistochemistry. Immunohistochemical analysis of hippocampal slices was performed as previously described (Kunkel et al., 1994). Rat hippocampi were dissected from appropriately aged animals, submerged in $4 \%$ paraformaldehyde for $48 \mathrm{hr}$, and cryoprotected in 30\% sucrose. Ten to 20- $\mu$ m-thick sections were cut using a Microm HM300 Universal Microtome Cryostat (Heidelberg) and mounted on subbed slides. For slices in which the immunostaining was visualized using diaminobenzidine (DAB), slices were treated sequentially with $0.05 \%, 0.1 \%$, and $0.3 \%$ $\mathrm{H}_{2} \mathrm{O}_{2}$ for 15 min to inhibit endogenous peroxidases. The slices were then treated with $50 \mu$ l of block solution ( $10 \%$ serum and $0.1 \%$ Triton X-100) for $30 \mathrm{~min}$ at room temperature, washed three times for $5 \mathrm{~min}$ each with PBS, and incubated with the primary antibody diluted in block for either $1 \mathrm{hr}$ at $37^{\circ} \mathrm{C}$ or overnight at $4^{\circ} \mathrm{C}$. The slices were washed three times for 5 min each with block and incubated with the secondary antibody for $1 \mathrm{hr}$ at $37^{\circ} \mathrm{C}$, then washed three times for $5 \mathrm{~min}$ each with PBS before incubating for $60 \mathrm{~min}$ at room temperature with avidin biotin complex (ABC) using the Vectastain Elite Kit (Vector Laboratories, Burlingame, $\mathrm{CA}$ ). The immunoreactivity was visualized by incubating the slices with $\mathrm{DAB}$ reagent according to the manufacturer's protocol (Sigma). Immunoreactivity visualized by immunofluorescence followed the same protocol excluding the endogenous peroxidase inhibition and $\mathrm{ABC} / \mathrm{DAB}$ incubation steps. Antibodies used in this study were anti-RPTP $\beta$ (1:10; BD Transduction Laboratories, San Diego, CA), $\beta$-tubulin (1:300; Sigma), and GFAP (1:200; ICN Biochemicals, Costa Mesa, CA or 1:1000; Sigma). 1D1 (1:500), 3H1 (1:10), and 3F8 (1:10) were purchased from the Developmental Biology Hybridoma Bank (University of Iowa, Iowa City, IA). Photomicrographs were obtained using a SPOT Digital Camera attached to a Nikon (Tokyo, Japan) Optiphot-2 Fluorescence Microscope.

Micro Ruby injections. The Micro Ruby injection solution was made by dissolving $1 \mathrm{mg}$ of Micro Ruby (Molecular Probes, Eugene, OR) in $10 \mu \mathrm{l}$ of $20 \mathrm{~mm}$ Tris Base followed by the addition of $50 \%$ glycerol (v/v). The Micro Ruby injection solution was loaded into a glass microelectrode made using Sutter Instruments (Novato, CA) glass tubing (BF-100-5810) pulled to a final tip diameter of $\sim 2 \mu \mathrm{m}$ using a Sutter P-97 electrode puller. The electrode was attached to a Leitz micromanipulator placed in a sterile hood. The tissue culture insert containing the hippocampal slice was transferred to a $100 \mathrm{~mm}$ sterile dish containing $10 \mathrm{ml}$ of growth medium under sterile conditions. An additional $1 \mathrm{ml}$ of growth medium was placed on top of the slice, and the $100 \mathrm{~mm}$ dish was placed on the stage of a Nikon TMS inverted microscope located in the sterile hood. The tip of the glass electrode was placed in the dentate granule cell layer of each hippocampal slice, and $\sim 0.1 \mu \mathrm{l}$ of the Micro Ruby solution was pressure injected into the slice. Forty-five minutes after injection of dye, the slices were fixed in $4 \%$ paraformaldehyde for $3 \mathrm{hr}$ at $4^{\circ} \mathrm{C}$ and mounted on microscope slides. Photomicrographs were obtained using either a SPOT Digital Camera attached to a Nikon Optiphot-2 Fluorescence Microscope or a Leica TCS SP2 Confocal microscope.

Timm stain. Timm stain recognizes the high concentration of zinc in mossy fiber terminals and is the most common method for identifying 
mossy fiber synapses. Timm stain was used to identify the presence of regenerated mossy fiber terminals using the previously published protocol from our laboratory (Nguyen et al., 1996). Briefly, cultures were treated with $1 \%$ sodium sulfide for $10 \mathrm{~min}$ and fixed in $70 \% \mathrm{ETOH}$ overnight at $4^{\circ} \mathrm{C}$. The cultures were rinsed with $0.1 \mathrm{M}$ PBS, mounted on gel-subbed microscope slides, and allowed to dry overnight. The cultures were exposed to the Timm staining solution for $30 \mathrm{~min}$ in the dark $(60 \mathrm{ml}$ of $50 \%$ gum arabic, $30 \mathrm{ml}$ of $5.7 \%$ hydroquinone, $10 \mathrm{ml}$ of $2 \mathrm{M}$ citrate buffer, and $500 \mu \mathrm{l}$ of $17 \%$ silver nitrate), rinsed in tap water and counterstained with neutral red.

Enzyme treatment of cultures. Chondroitin ABC lyase, keratanase I, and keratanase II (Seikagaku America, East Falmouth, MA) were dissolved in HBSS to a final concentration of $10 \mathrm{U} / \mathrm{ml}$ for $\mathrm{ABC}$ lyase and $1 \mathrm{U} / \mathrm{ml}$ for keratanases I and II. All cultures treated with enzymes were interface slice cultures or dentate explants in which the surface of the cultured tissue was exposed to air. Because the cultured tissue was not submerged, $1 \mu \mathrm{l}$ of enzyme solution could be applied directly to the surface of the culture every $24 \mathrm{hr}$. When cultures were treated with two or three enzymes, all enzymes were combined together so that all enzymes could be applied with a single $1 \mu$ application. For experiments using intact hippocampal slices, the cultures were treated $2-5 \mathrm{~d}$ before mossy fiber transection. Dentate explants were treated with enzymes for $2 \mathrm{~d}$ starting on the day of culture.

Western analysis. To characterize protein expression in cultured cells, samples grown on filter inserts were harvested, subjected to SDS-PAGE, immunoblotting, and signal detection with ECL (Amersham Biosciences, Piscataway, NJ). Equal protein was loaded in each lane (3-10 $\mu \mathrm{g})$. Primary antibodies used were: 4S (1:1000; Seikagaku America), 3H1 and 3F8 (1:10; Developmental Hybridoma Bank), and antiphosphotyrosine antibody (1:1000; Santa Cruz Biotechnology, Santa Cruz, CA). Rabbit anti-mouse-HRP-linked secondary antibodies (Sigma) were used at 1:10,000 dilutions. Specific primary and secondary antibody combinations are indicated in the figure legends. Films were scanned using an HP Scanjet 6300, and the densities of the specific bands were analyzed using Eastman Kodak (Rochester, NY) 1D gel analysis software. Sample values are expressed as arbitrary units relative to the value of the most dense band.

Statistical analysis. All statistical analyses were performed using SigmaStat version 3.0 and plotted using SigmaPlot version 8.0.

\section{Results}

\section{Distribution of proteoglycans in hippocampus}

The goal of this study was to examine whether neurocan and phosphacan are involved in directing MF outgrowth and regeneration in the hippocampus. The MF pathway exhibits robust outgrowth in rodents during the first postnatal week. We therefore examined the role of neurocan and phosphacan in regulating MF outgrowth using cultured hippocampal slices from postnatal day 4 (P4)-P7 rats. Cultured hippocampal slices provide a powerful model for studying MF outgrowth because they exhibit much of the anatomy and circuitry of the hippocampus in intact animals. The CA1 and CA3 pyramidal cell layers, as well as the granule cell layer of the dentate gyrus (DG), were clearly observed in slices made from $\mathrm{P} 4$ rats cultured for $7 \mathrm{~d}$ in vitro (Fig. $1 \mathrm{~A}$ ). In addition, MFs and their synaptic terminals in cultured hippocampal slices were restricted to the SL (Fig. $1 C, B$, respectively) just as they are in intact hippocampus.

The 1D1 antibody was used to analyze the immunohistochemical distribution of neurocan in P7 rat hippocampus (Fig. 2A). 1D1 immunostaining in the stratum lucidum (SL) of P7 rats was much lower than in adjacent stratum radiatum (SR) (Fig. 2A). This is significant because high levels of CSPG in SR could act as an inhibitory boundary (arrow) that prevents MFs from migrating outside of the SL. This pattern is also very similar to that previously reported using the IG2 antibodies to identify neurocan (Kurazono et al., 2001). Extracellular phosphacan immu-
A) Nissl stain

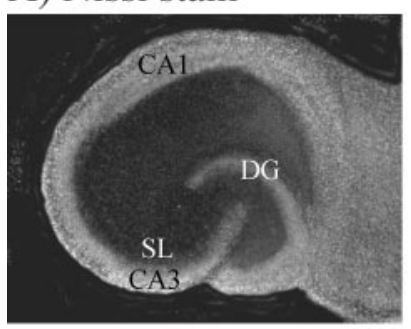

C) Micro Ruby

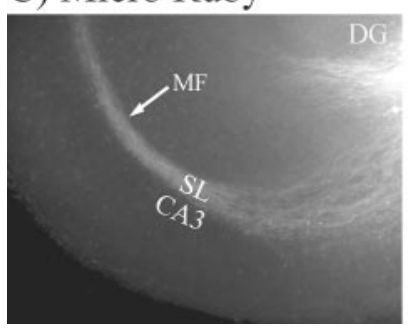

B) Timm stain

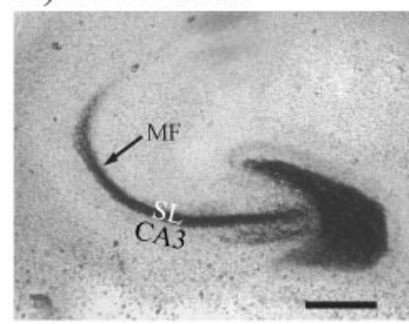

Figure 1. Mossy fibers (MFs) exhibited their normal distribution within stratum lucidum(SL) in hippocampal slice cultures maintained in culture for $7 \mathrm{~d}$. $A$, The cultured slices were stained with fluorescent Nissl stain to demonstrate the presence of the CA1 and CA3 pyramidal cell layers and the granule cell layer in the dentate gyrus (DG). B, MF presynaptic terminals were stained black using Timm stain (black arrow), which recognizes the high concentration of zinc packaged in MF synaptic vesicles. The black band that represents the MF terminals also defines the SL, which is a narrow band located just above the CA3 pyramidal cell bodies. Thus, the SL and the MF terminal field form a precise banding pattern that lies over the proximal apical dendrites of CA3 pyramidal neurons but stop at the border between areas CA3 and CA1. C, This same precise banding pattern within SL can also be seen when MF axons (white arrow) were visualized by stereotaxically injecting fluorescent Micro Ruby dye into the granule cell layer of living cultures. Scale bars: $A, B, 500 \mu \mathrm{m} ; C, 200 \mu \mathrm{m}$.

nostaining was performed using the 3F8 (Fig. 2 B) and 3H1 (Fig. $2 D)$ antibodies, which recognize phosphacan containing either CS-GAGs (phosphacan) or KS-GAGs (KS-phosphacan), respectively. 3F8 staining exhibited an even distribution throughout all hippocampal layers, whereas $3 \mathrm{H} 1$ showed very light staining in all strata proximal to the dentate (Fig. $2 D$, arrowhead). The lack of $3 \mathrm{H} 1$-immunostaining proximal to the dentate suggests that extracellular KS-phosphacan is not in the proper location to direct growing MFs into the SL. The granule cell layer also exhibited low levels of KS-phosphacan staining (Fig. $2 D$, boxed area is shown at higher magnification in Fig. $2 F$ ). We also examined the distribution of the receptor protein tyrosine phosphatase $\beta / \zeta(\operatorname{RPTP} \beta / \zeta)$, the transmembrane form of phosphacan. RPTP $\beta / \zeta$ staining is evenly distributed throughout all layers of CA3 and the dentate (Fig. 2C). The full-length form of RPTP $\beta / \zeta$ also contains KSGAGs but is expressed at a much lower level than extracellular phosphacan (Nishiwaki et al., 1998). RPTP $\beta / \zeta$ staining therefore appears to be a subset of phosphacan staining. Thus, we will compare the relative patterns of $3 \mathrm{H} 1$ and $\mathrm{RPTP} \beta / \zeta$ immunostaining. In contrast to $3 \mathrm{H} 1$ staining, $\mathrm{RPTP} \beta / \zeta$ staining was more apparent around the CA3 pyramidal and granule cell bodies (boxed areas of DG in Fig. 2C,D are shown at higher magnification in Fig. $2 E, F)$. Thus, relative to $3 \mathrm{H} 1$, more of the RPTP $\beta / \zeta$ staining is localized between the cell bodies of dentate granule cells and CA3 pyramidal cells. The difference in relative distribution of RPTP $\beta / \zeta$ and extracellular KS-phosphacan may signify an important difference in their function. In this regard, it is interesting to note that RPTP $\beta / \zeta$ is most highly expressed during the maximal time of MF outgrowth and the dentate gyrus is one of three brain areas that continue to express RPTP $\beta / \zeta$ in adult brain (Levy et al., 1993; Nishiwaki et al., 1998). 
A) Neurocan (1D1)

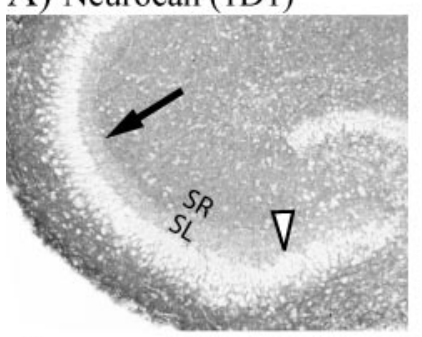

C) RPTP/Phosphacan

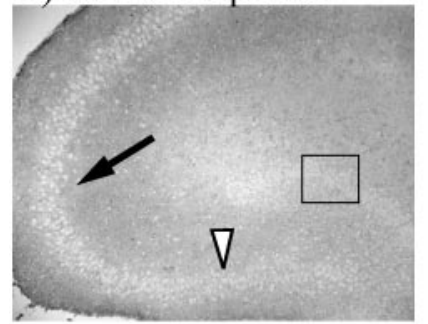

E) RPTP/Phosphacan

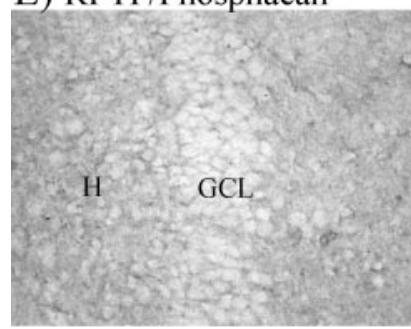

B) Phosphacan (3F8)

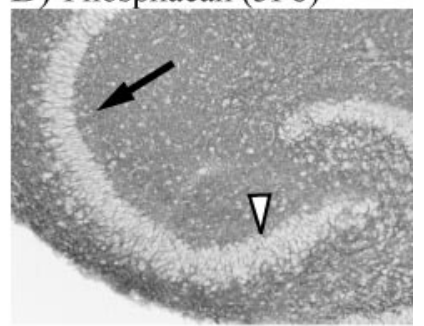

D) KS-Phosphacan (3H1)

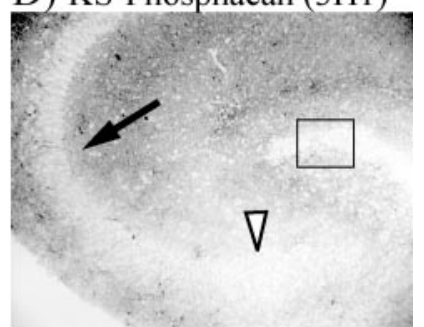

F) KS-Phosphacan (3H1)

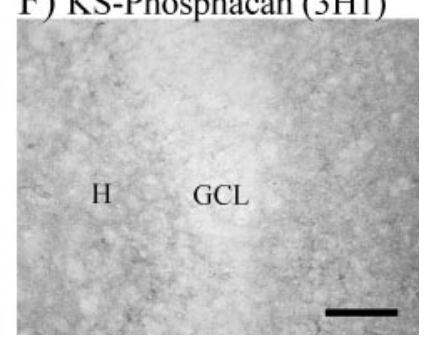

Figure 2. Distribution of neurocan and three forms of phosphacan in cryostat sections from P7 rat hippocampus. $A$, Neurocan immunostaining was lower in the MF termination zone (SL) compared with stratum radiatum (SR). Thus, neurocan appears to form an inhibitory CSPG border (arrow) that could inhibit MFs in SL from growing into SR. The CA3 pyramidal cell layer also showed light staining (arrowhead). $B-F$, Three different antibodies were used to stain the different types of phosphacan. $B$, Extracellular phosphacan containing only CS-GAGs was stained using 3F8 antibody, which showed an even distribution throughout all layers of the hippocampus. C, RPTP $\beta / \zeta$ immunostaining (cytosolic epitope) also showed even staining throughout the hippocampus. Note that the intensity of RPTP $\beta / \zeta$ staining was enhanced using Photoshop to facilitate comparisons with the other two forms of phosphacan. D, Phosphacan containing extracellular KS-GAGs was stained using the $3 \mathrm{H} 1$ antibody which showed relatively low staining throughout all areas of $C A 3$ proximal to the $D G$, including the $C A 3$ pyramidal cell layer (arrowhead). Note that RPTP $\beta / \zeta$ is strongly distributed between neuronal cell bodies, whereas $3 \mathrm{H} 1$-immunoreactive extracellular KS-phosphacan is only weakly distributed between the cell bodies (boxed areas in (and D shown at higher magnification in $E$ and $F$ ). GCL, Granule cell layer; $H$, hilus. Scale bars: $A-D, 200 \mu \mathrm{m} ; E, F, 50 \mu \mathrm{m}$.

The immunohistochemical distribution of phosphacan in intact hippocampal sections could not distinguish whether phosphacan and RPTP $\beta / \zeta$ were expressed by astrocytes or granule cell bodies (Fig. $2 E, F$ ). We, therefore, dissected the granule cell layer away from the other neuronal cell layers of the hippocampus, dissociated the cells and maintained them in culture for 5-6 d (Figs. 3, 4). In Figure 3, the dissociated cultures were stained using 3F8 (Fig. 3A) or anti-RPTP $\beta / \zeta$ (Fig. 3B) antibodies in combination with anti-GFAP antibodies to label astrocytes (Fig. $3 C, D)$. When the red and green images were merged, all of the 3F8- and RPTP $\beta / \zeta$-positive cells were co-labeled by GFAP (Fig. $3 E, F)$. However, when these cultures were stained with $3 \mathrm{~F} 8$ and antibodies against $\beta$-tubulin to label neurons (Fig. 4), neuronal cells and their processes did not appear to express phosphacan, suggesting that the $3 \mathrm{~F} 8$ and RPTP $\beta / \zeta$ immunostaining observed

A) $3 \mathrm{~F} 8$

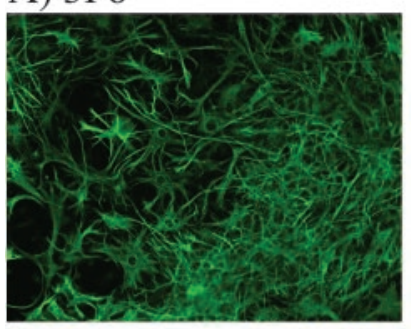

C) GFAP

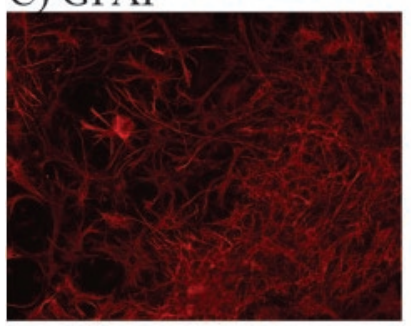

E) Merged

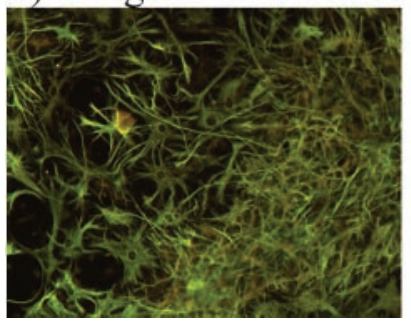

B)RPTP

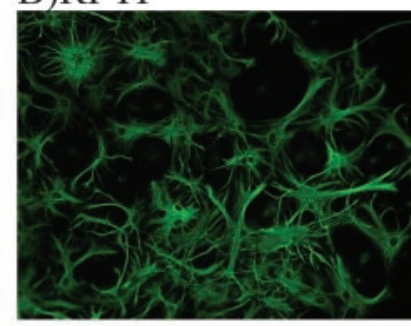

D) GFAP

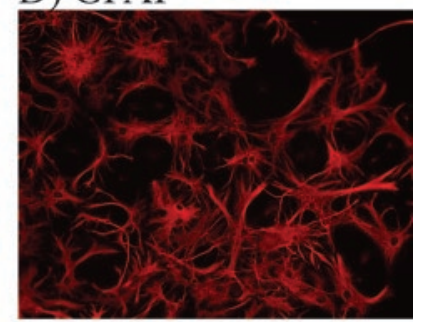

F) Merged

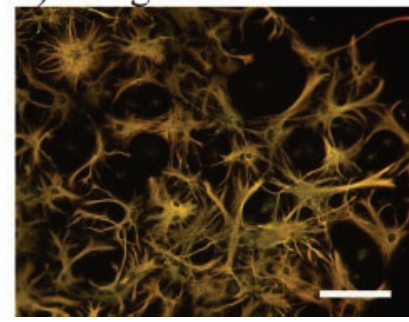

Figure 3. RPTP $\beta / \zeta$ and extracellular phosphacan are expressed by astrocytes in dissociated DG cultures. Dissociated DG cultures were stained green using 3F8 $(A)$ and anti-RPTP $\beta / \zeta$ antibodies $(B)$. Astrocytes in both sets of cultures were stained red with anti-GFAP antibody $(C, D)$. The red and green merged images demonstrated that both extracellular phosphacan and $\operatorname{RPTP} \beta / \zeta$ were expressed by astrocytes (yellow staining, $E$ and $F$ ). The same results were obtained in cultures stained for $3 \mathrm{H} 1$ and GFAP (data not shown). Scale bar, $100 \mu \mathrm{m}$.

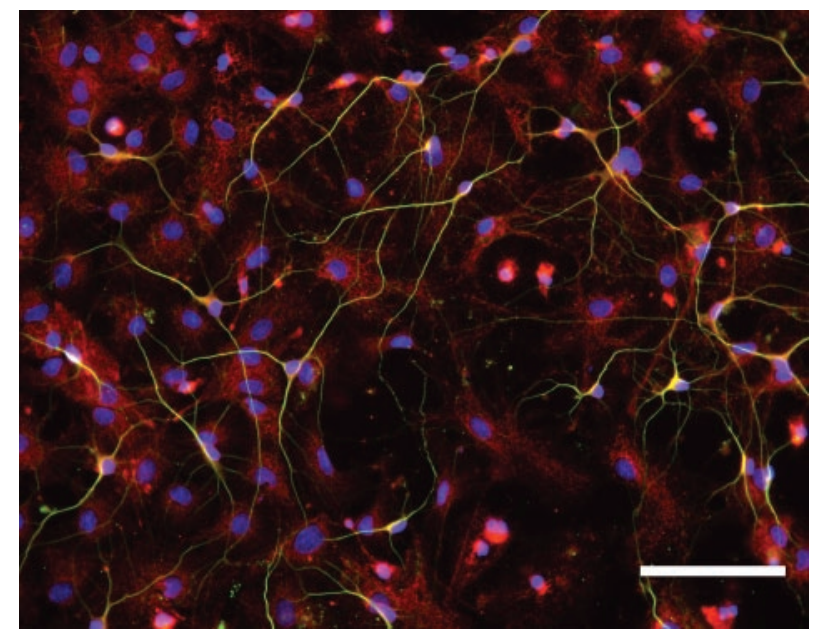

Figure 4. Neurons in dissociated DG cultures do not express phosphacan. Neurons in the same group of dissociated DG cultures shown in Figure 3 were stained green using anti- $\beta$ tubulin antibodies. Extracellular phosphacan in the cultures was stained red using the 3F8 antibody. Nuclei were stained blue using DAPI. The merged image shows little yellow, indicating very limited overlap between 3F8 and $\beta$-tubulin staining. Scale bar, $200 \mu \mathrm{m}$.

in Figure 2 primarily represented staining of astrocytic processes located between neuronal cell bodies. These data are in agreement with previous reports that phosphacan is made by astrocytes (Rauch et al., 1991; Canoll et al., 1993). 

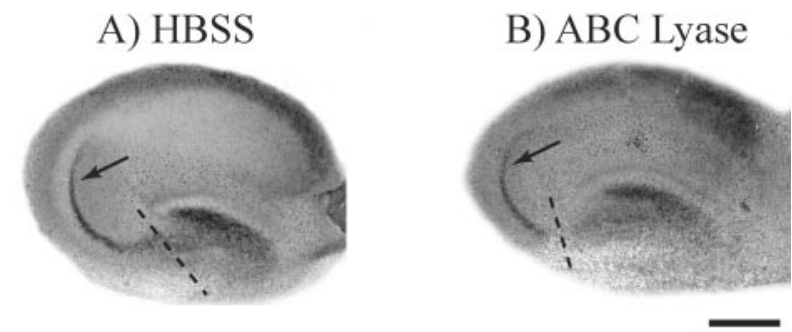

Figure 5. Chondroitin $A B C$ lyase does not alter MF regeneration. Cultured hippocampal slices were treated with chondroitin $A B C$ lyase to enzymatically digest CS-GAGs, and control slices were treated with HBSS. MFs in these two groups of slices were then transected (dashed lines) and allowed to regenerate for $5 \mathrm{~d}$. Timm stain was used to determine whether MFs regenerated their normal synaptic pattern in $\mathrm{SL}$ as shown in Figure $1 B$. The data show that cultures treated with $\operatorname{HBSS}(A)$ and those treated with chondroitin $A B C$ lyase $(B)$ both regenerated their normal synaptic pattern within SL (arrows). Scale bar, $500 \mu \mathrm{m}$.

Table 1. Keratanase disrupts the lateral organization of MFs in hippocampal slices

\begin{tabular}{lcc}
\hline Treatment & Cross section $(\mu \mathrm{m})$ & Significance \\
\hline HBSS control $(n=24)$ & $99.25 \pm 8.49$ & \\
$\mathrm{ABC}(n=5)$ & $104 \pm 22.47$ & $p<0.977$ \\
$\mathrm{ABC}+\mathrm{K} 1+\mathrm{K} 2(n=20)$ & $319.35 \pm 24.19$ & ${ }^{*} p<0.001$ \\
$\mathrm{~K} 1+\mathrm{K} 2(n=3)$ & $341.67 \pm 40.99$ & ${ }^{*} p<0.006$ \\
K1 $(n=5)$ & $303 \pm 24.54$ & ${ }^{*} p<0.001$ \\
K2 $(n=4)$ & $221 \pm 46.19$ & ${ }^{*} p<0.008$ \\
Total keratanase $(n=32)$ & $306.59 \pm 17.57$ & ${ }^{*} p<0.001$ \\
\hline
\end{tabular}

The regeneration of MFs was analyzed in cultured rat hippocampal slices that were treated with chondroitin $A B C$ lyase (ABC), keratanase $1(K 1)$, or keratanase 2 (K2) as described in Materials and Methods. Regenerating MFs normally form a narrow band with a cross section that is $\sim 100 \mu \mathrm{m}$ wide. MFs were stained using Micro Ruby injections (see Materials and Methods), and fluorescent images were obtained using a Spot Digital Camera. The line tool in the Spot Camera software was calibrated using an image of a micrometer, and a line was drawn across the width of the regenerated MF pathway. Cultures treated with either K1 or K2 alone or in combination with ABC or each other produced a statistically significant increase in the cross section of regenerated MFs compared with MFs in control cultures treated with HBSS. However, ABC treatment alone produced no significant increase in cross section versus HBSS control. These results demonstrate that MFs in keratanase-treated slices regenerated in a highly disorganized manner. Mann-Whitney rank sum statistical analysis was performed using SigmaStat 3.0 software. Asterisks mark enzyme treatments that are significantly different from HBSS control.

Function of proteoglycans in MF outgrowth and regeneration Functional studies were performed using chondroitin ABC lyase treatment of cultured slices to digest and inactivate the functions of neurocan and phosphacan regulated by CS-GAGs. Keratanases I and II were used to inactivate the functions of phosphacan regulated by KS-GAGs. Based on the anatomical distribution of proteoglycans in the hippocampus, we predicted that chondroitin ABC lyase would have the biggest influence on MF outgrowth and regeneration while keratanase would produce little or no change in MF behavior.

First we examined whether enzymatic removal of CS-GAGs from neurocan and phosphacan affected the pattern of MF outgrowth and regeneration in cultured hippocampal slices. Approximately $1 \mu \mathrm{l}$ of chondroitin ABC lyase was applied directly to the surface of the cultured slice each morning while an equal volume of HBSS was applied to control cultures. After the first $2 \mathrm{~d}$ of enzyme treatment, the MFs were transected and allowed to regenerate for $5 \mathrm{~d}$. The cultures were treated with enzyme every $24 \mathrm{hr}$ after MF transection to ensure the continued removal of inhibitory CS-GAGs. Timm-stained cultures demonstrate that the pattern of MF regeneration in chondroitin $\mathrm{ABC}$ lyase-treated slice cultures (22 of 23 slices) was not different from control cultures treated with vehicle alone (Fig. 5, arrows). The same results were observed when chondroitin $\mathrm{ABC}$ lyase-treated slices were labeled using Micro Ruby dextran dye injections to identify MF axons (Table 1, Fig. 6, top row). Photomicrographs of chondroitin $\mathrm{ABC}$ lyase treated cultures clearly reveal that regenerating
MFs remain localized to the stratum lucidum (arrows) similar to untreated and uncut MFs (Fig. $1 B, C$ ).

Surprisingly, regenerating MFs in cultures treated with keratanase were extremely disorganized and ran laterally throughout strata oriens, radiatum, and pyramidale of area CA3 (Table 1, Fig. 6 , bottom row). This disorganized pattern was observed when cultured slices were treated with chondroitin $\mathrm{ABC}$ lyase plus keratanases I and II $(\mathrm{ABC}+\mathrm{K} 1+\mathrm{K} 2)$, keratanase I alone $(\mathrm{K} 1)$, keratanase II alone (K2), or a combination of keratanases I and II $(\mathrm{K} 1+\mathrm{K} 2)$ (Table 1). Thus, the presence of keratanase was strongly correlated with disruption of MF regeneration (total number of cultures treated with keratanase $=32$ ). In contrast, the pattern of MF regeneration after treatment with $\mathrm{ABC}$ lyase was not statistically different from control cultures (Table 1). Regenerating MFs also appeared to be spread over a larger vertical area of the hippocampal slices, although these data were not quantified (Fig. 6). Importantly, the pattern observed after keratanase treatment did not resemble the failure to regenerate that was observed in a previous study after inhibition of laminin expression (Grimpe et al., 2002). In this previous study, MFs formed normal bundles of fibers in the SL but failed to extend beyond the point of transection when laminin expression was inhibited. In the present study, however, MFs grew well past the point of transection but failed to coalesce into fasciculated bundles within the SL. Most importantly, they exited the DG in areas outside the SL and continued to grow throughout all strata of area CA3 in a disorganized manner. The fact that these MFs were not restricted to parallel bundles within SL made it difficult to measure their exact length of regeneration. However, numerous MFs were observed in area CA3A, which is the normal termination point of the MF pathway.

The fact that neurocan and CS-phosphacan are strongly expressed in and around the synaptic target region of MFs makes it difficult to understand how MFs could be unaffected by chondroitin ABC lyase treatment. It is equally difficult to understand why MFs were so dramatically affected by keratanase treatment because KS-phosphacan is not strongly expressed in CA3 proximal to the DG. There are four potential explanations for these results. First, it is possible that our chondroitin $\mathrm{ABC}$ lyase treatment protocol was ineffective at removing CS-GAGs from living hippocampal slices. Second, it is possible that MFs are unusual in that they are inhibited by KSPGs but not CSPGs. Third, it is possible that KS containing RPTP $\beta / \zeta$ located on astrocytic process between the granule and pyramidal cell bodies directly modifies astrocyte behavior in a manner that changes MF outgrowth. Fourth, it is possible that the loss of KS on extracellular phosphacan could alter cell-cell or cell-matrix interactions that indirectly modify astrocyte behavior and subsequently change MF outgrowth.

To eliminate the first possible explanation, we used Western analysis to confirm that the enzymatic treatment used in our study effectively removed GAG sugars from proteoglycans in cultured hippocampal slices. Western analysis was performed on homogenates from HBSS (control) and chondroitin ABC lyasetreated cultures using the $4 \mathrm{~S}$ antibody. The $4 \mathrm{~S}$ antibody only recognizes CSPGs after they have been digested with chondroitin ABC lyase. A 4S-positive band was only observed in homogenates from living cultures that had been treated with chondroitin $\mathrm{ABC}$ lyase (A) but was completely absent in homogenates from cultures treated with HBSS (H) (Fig. 7, row 1, lanes 1 and 2). These data suggest that most of the CSPG in living cultures was enzymatically digested using our method of applying $\mathrm{ABC}$ lyase to living slice cultures. 

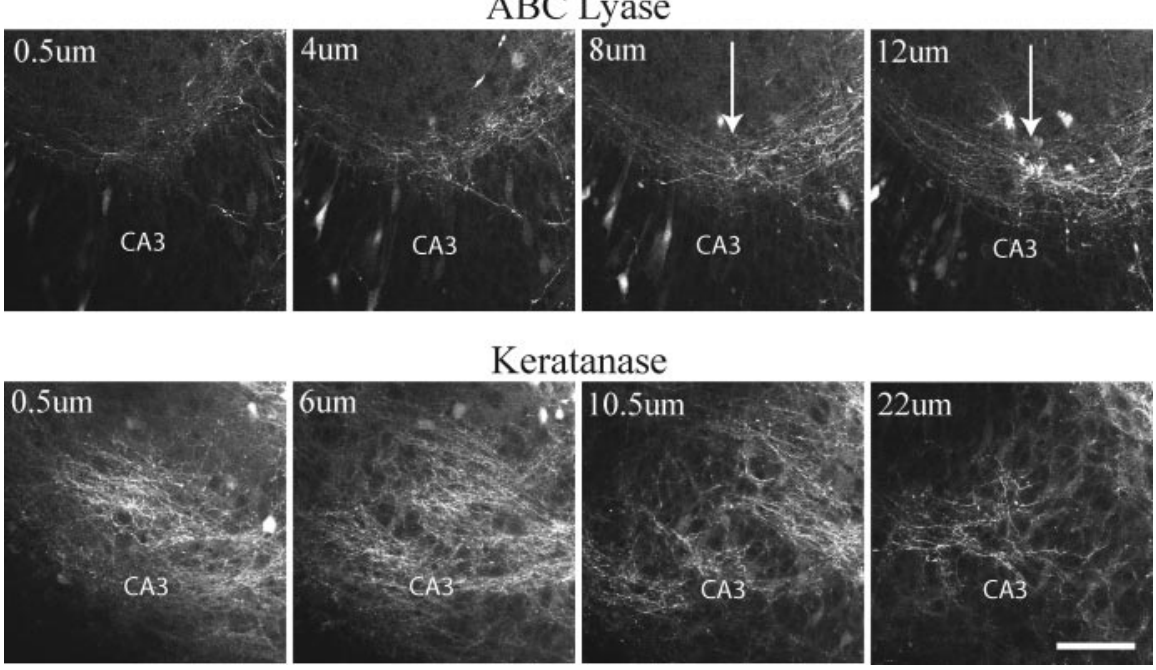

Keratanase
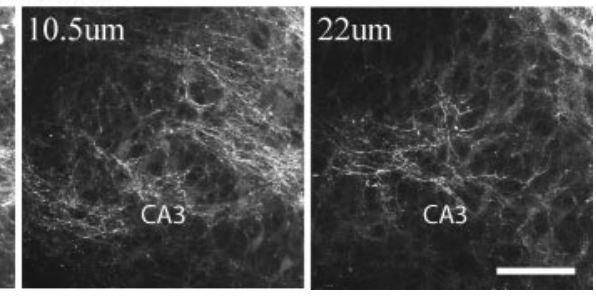

Figure 6. Confocal images show that MFs regenerated in a disorganized manner when hippocampal slice cultures were treated with keratanase. Slices were treated with either chondroitin ABC lyase or keratanase II. MFs were stained by injecting Micro Ruby into the granule cell layer of living slices. A series of 0.5 - $\mu \mathrm{m}$-thick optical sections were taken through the slices using a Leica TCS SP2 confocal microscope. Whereas MFs regenerated their normal pattern within the SL in ABC lyase-treated slices (arrows), MFs in keratanase-treated slices wandered laterally into stratum pyramidale and stratum radiatum. MFs in keratanase-treated cultures also appeared to spread more in the vertical plane of the cultures. Scale bar, $100 \mu \mathrm{m}$.

A similar analysis was performed on cultured slices treated with keratanases I and II $(\mathrm{K} 1+\mathrm{K} 2)$ to determine whether these enzymes were effective in removing KS-GAGs from phosphacan. These analyses were performed using the $3 \mathrm{H} 1$ antibody, which specifically recognizes the KS epitope on phosphacan. $3 \mathrm{H} 1 \mathrm{im}-$ munoblot analysis showed a complete loss of keratan sulfateimmunoreactive band in homogenates from slices treated with keratanases I and II (K) (Fig. 7, row 2, lanes 3 and 4). When the same blot was stripped and reprobed with 3F8 antibody, there was no significant difference between the average intensity of the bands from slices treated with HBSS versus those treated with keratanases I and II (Fig. 7, row 3), which suggests that keratanase treatment does not result in nonspecific degradation of CSphosphacan. Thus, treatment of cultured hippocampal slices with $\mathrm{ABC}$ lyase and keratanase efficiently and specifically remove CS and KS-GAG sugars, respectively.

To investigate our second possible explanation that MFs are unusual in that they are inhibited by KSPGs but not CSPGs, we examined how a relatively pure population of actively growing MFs responded when they came into contact with CSPGs or KSPGs. For these experiments, the DG cell layer was surgically isolated as previously described for dissociated DG cultures except that the DGs were left as intact explants rather than being dissociated into individual cells. Half of the culture substrate was coated with laminin, and the other half was coated with a mixture of CSPGs and KSPGs isolated from embryonic chicken brain (Chemicon). The Chemicon proteoglycan mixture, which included neurocan, phosphacan, versican, and aggrecan, is similar to the composition of proteoglycans found in rat brain (Rauch et al., 1991; Oohira et al., 1994; Meyer-Puttlitz et al., 1995; Milev et al., 1998). When MFs growing on laminin contacted the proteoglycan border, the majority of MFs turned sharply to avoid it (Fig. $8 \mathrm{~A}$, arrowhead). In some cases, the explants overlapped both the laminin and $1 \times$ proteoglycan substrates. In these cases, MFs exhibited some ability to adapt and grow on the $1 \times$ proteoglycans substrate (Fig. $8 B, F$, arrows), as previously described for dorsal root ganglion neurons (Snow and Letourneau, 1992; Condic et al., 1999). However, MFs still made an abrupt turn when they reached the $2 \times$ proteoglycans substrate (Fig. $8 B, F$, arrowheads). Thus, most MFs turned to avoid a proteoglycan step gradient. These results suggest that when tested using similar experimental paradigms, MFs are inhibited by proteoglycans in a manner that is similar to other types of axons. Indeed, MF outgrowth was reportedly inhibited when they came into contact with phosphacan applied to the culture substrate in a manner similar to our in vitro system (Wu et al., 2000).

The fact that the proteoglycan mixture contained both neurocan and phosphacan made it necessary to test whether MF inhibition was mediated by CS or KS proteoglycans. When the proteoglycan substrate was treated with chondroitin ABC lyase to enzymatically remove CS-GAGs before plating of DG explants, MFs freely crossed the CSPG substrate (Fig. 8C,G, arrows). These observations are consistent with other studies showing that chondroitin ABC lyase relieves inhibition of axon outgrowth by CSPGs (Snow et al., 1990, 1991; Brittis et al., 1992). To examine whether the inhibitory properties of the proteoglycan mixture were attributable to KS-GAGs on phosphacan, we pretreated the substrate with a mixture of keratanases I plus II (K1K2). In contrast to chondroitin ABC lyase treatment of the substrate, MFs were still strongly inhibited after keratanase treatment (Fig. 8D,H, arrowhead). MFs originating on the $1 \times$ proteoglycan substrate were unable to cross the $2 \times$ step gradient (arrowhead) in the same manner as MFs growing on the untreated proteoglycan substrate shown in Figure 8, $B$ and $F$. These results obtained using the Chemicon proteoglycan mixture suggest that proteoglycans containing CS-GAGs are primarily responsible for inhibition of MF outgrowth compared with the inhibition produced by proteoglycans containing KS-GAGs. The conclusion that KSPGs have little affect on MF outgrowth is further supported by the fact that MF outgrowth was not inhibited from growing on a substrate made from KS-GAGs purified from bovine cornea (Seikagaku Corp., data not shown).

Although the results shown in Figure 8 appear to contradict those shown in Figures 5 and 6, the combined results in these figures actually help to clarify how MF outgrowth is regulated in their native environment. In their native environment of the hippocampus, MFs must navigate through the extracellular matrix where CSPGs are located in and around their synaptic area in CA3. The painting of proteoglycans on tissue culture substrates as shown in Figure 8 represents the experimental model system that has typically been used to examine how growth cones behave when they encounter specific guidance molecules. Therefore, the fact that chondroitin ABC lyase dramatically alters the behavior of MFs on the proteoglycan substrate in Figure 8, whereas keratanase does not, suggests that extracellular proteoglycans in CA3 are unlikely to be responsible for the misrouting of MFs observed in cultured hippocampal slices after keratanase treatment. This apparent contradiction between the results observed in cultured slices and explants grown on proteoglycan substrates may be resolved if our third possible explanation is considered. The third explanation suggests that KS-containing RPTP $\beta / \zeta$ located be- 

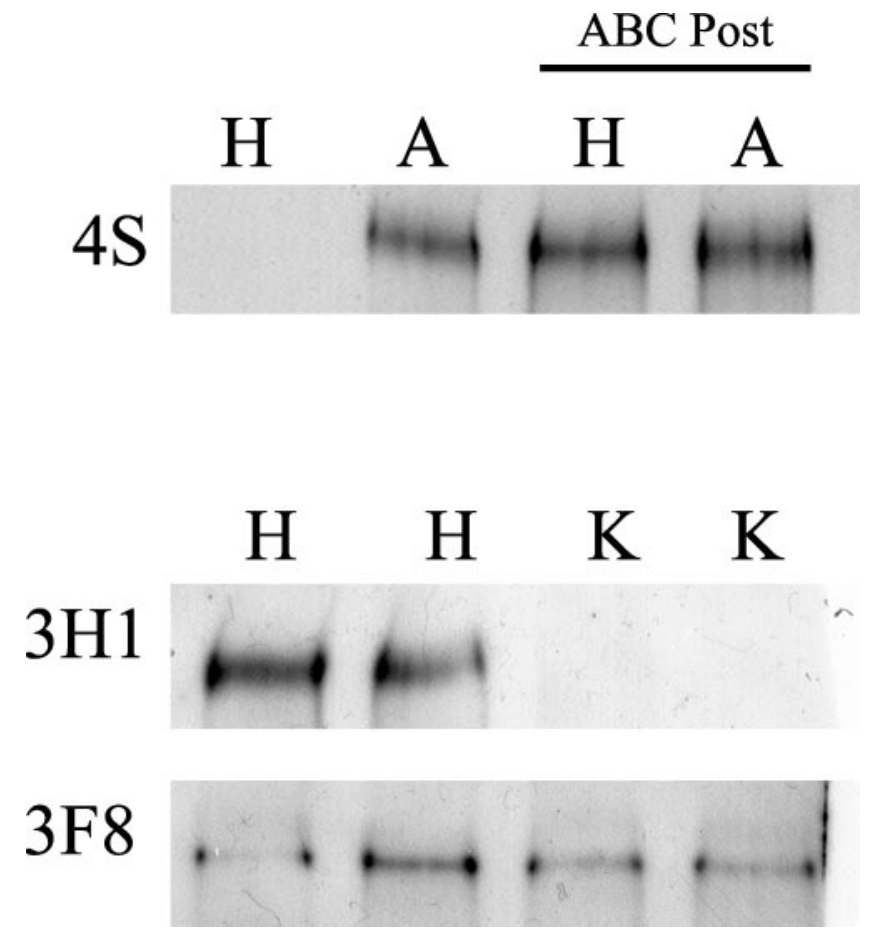

Figure 7. Western analysis demonstrated that chondroitin $A B C$ lyase and keratanase treatments were effective at removing $C S$ - and $K S$-GAG sugars from proteoglycans in living hippocampal cultures. Hippocampal cultures were treated with either HBSS $(\mathrm{H}), \mathrm{ABC}$ lyase $(\mathrm{A})$, or a mixture of keratanases I+II (K). First, Western analysis was performed using the $4 S$ antibody, which only recognizes CSPGs after ABC lyase has removed CS-GAGs but does not recognize intact CSPGs. The 4S Western blot showed no staining in H-treated cultures and a strong band in the A-treated cultures $(n=3)$. To demonstrate that there were equal amounts of 4S-positive material in the homogenates of $\mathrm{H}$ - and A-treated cultures, aliquots of the same homogenates run in lanes 1 and 2 were post-treated with $A B C$ lyase (ABC Post). The 4S-positve band from $\mathrm{H}$-treated cultures was not significantly different from the band in A-treated cultures when the homogenates were post-treated with $\mathrm{ABC}$ lyase ( $n=3$, last two lanes). To test the effectiveness of keratanase treatment at removing KS-GAGs from phosphacan, Western analysis was performed on homogenates from slice cultures treated with either $\mathrm{H}$ or $\mathrm{K}$. Because the $3 \mathrm{H} 1$ antibody recognizes KS-GAGs on phosphacan, the absence of a 3H1-positive band in homogenates from K-treated cultures demonstrated that our protocol was effective at removing KS-GAGs from phosphacan. The same blot was then stripped and reprobed for $3 \mathrm{~F} 8$, which recognizes phosphacan without KS-GAGs. The intensity of the 3F8-positive band from K-treated cultures was not significantly different from those from $\mathrm{H}$-treated cultures $(n=3)$, which demonstrates that keratanase treatment did not degrade non-KS forms of phosphacan.

tween the granule cell bodies has a bigger influence on MF outgrowth than do extracellular CS-proteoglycans in the synaptic target area. If this explanation is correct, then treatment of DG explants with keratanase should produce a change in the phosphorylation of specific proteins. When dentate explants were treated with $\mathrm{K} 1 \mathrm{~K} 2$, Western analysis demonstrated that this treatment reduced the phosphorylation of an $80 \mathrm{kDa}$ band in homogenates from treated DG explants (Fig. 9). Similar changes in protein phosphorylation were not observed in dentate explants treated with $\mathrm{ABC}$ lyase (data not shown).

These data appear to support the conclusion that removal of KS-GAGs results in an increase in activity of RPTP $\beta / \zeta$. Because $\mathrm{RPTP} \beta / \zeta$ is expressed on astrocytes, the primary affect of keratanase should be to change the behavior of astrocytes. The large size and thickness of hippocampal slice cultures makes it difficult to observe astrocyte behavior within these slices. We therefore cultured small explants from either the dentate or CA3 regions and analyzed astrocyte migration on a laminin substrate after enzyme
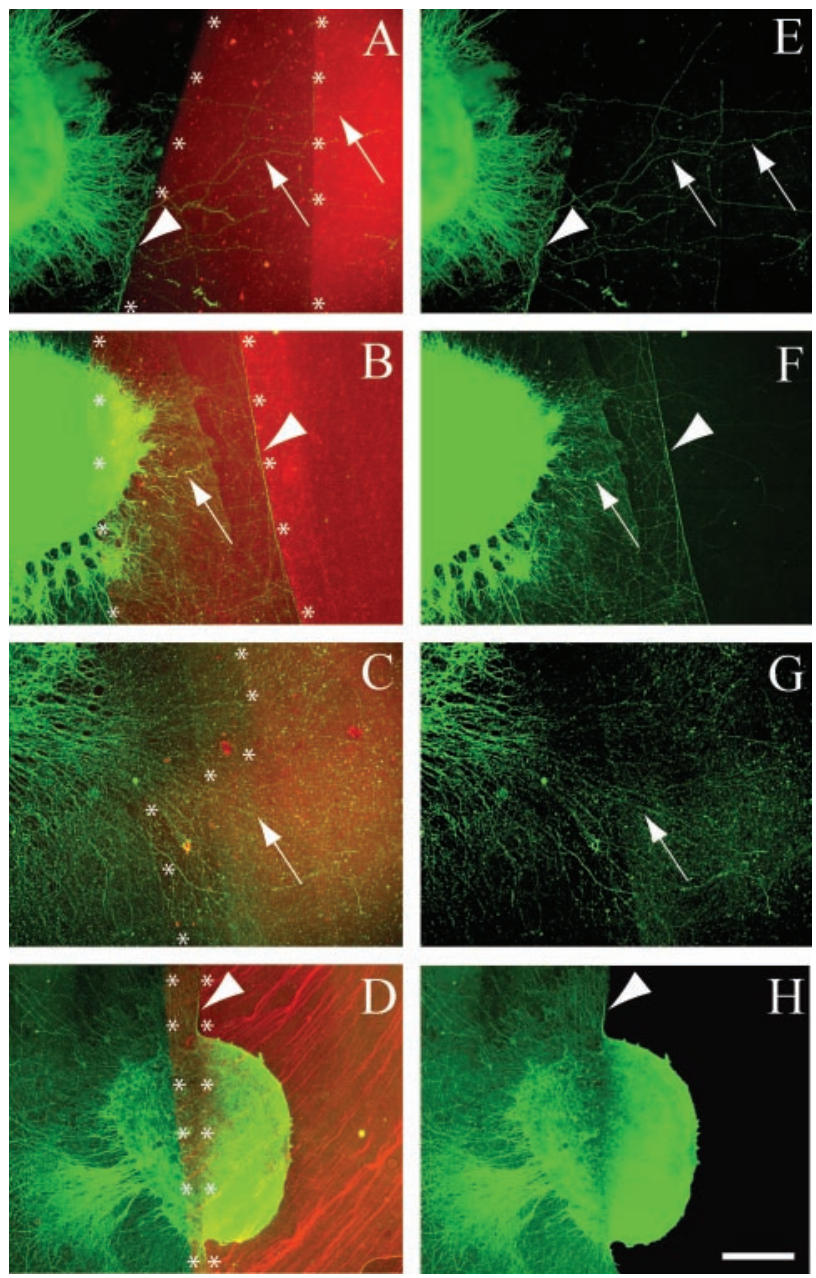

Figure 8. MF outgrowth was inhibited by contact with CSPG but not KSPG substrates. DG explants were placed on a laminin substrate next to an area on which a mixture of brain proteoglycans was applied. Axons were stained green using anti- $\beta$-tubulin immunohistochemistry. Alexa 594 was added to the proteoglycan mixture to mark its location (red fluorescence). Two coats of the proteoglycan mixture were applied in a partially overlapping manner (see Materials and Methods), resulting in areas that contained 1 layer of proteoglycan $(1 \times)$ and areas that contain two layers of proteoglycan $(2 \times)$. The laminin and $1 \times$ proteoglycan border is marked by asterisks, as is the border between $1 \times$ and $2 X$ proteoglycan substrates. The red and green images were merged to visualize how MF axons behaved when they encountered a proteoglycan border (asterisks). $E-H$ are the same figures as $A-D$ except that only the green $\beta$-tubulin staining is shown to facilitate observation of axons. Arrows and arrowheads identify the same axon segments in paired figures. Arrowheads identify axons that were inhibited by the proteoglycans substrate, whereas arrows identify axons that were not inhibited. $A, E$, When given a choice between laminin and proteoglycans, most MFs turned abruptly to avoid the proteoglycan mixture $(n=13) . B, F$, When $D G$ explants were placed on a $1 \times$ concentration of proteoglycan, MFs extended onto the lower concentration (arrow) but turned to avoid a $2 \times$ step gradient (arrowhead, $n=22$ ). In ( and $G$, the same proteoglycan substrate was treated with chondroitin $A B C$ lyase ( $10 \mathrm{U} / \mathrm{ml}$ for $3 \mathrm{hr}$ ) before plating DG explants. The substrate was washed to remove any remaining enzyme before plating the $D G$ explants. In contrast to the results observed in $A$, MFs readily grew across the $A B C$ lyase treated proteoglycans substrate in 38 of 43 cultures. Note that it was necessary to show images $C$ and $G$ at higher magnification to clearly observe axons that grow in a less fasciculated manner on the CSPG substrate that has been treated with $A B C$ lyase. $D, H$, However, when the proteoglycan substrate was treated with the same concentration of keratanases I+II that removed KS-GAGs from cultured hippocampal slices (1 U/ml for $3 \mathrm{hr}$ ) (Fig. 7), MF outgrowth was still inhibited by the proteoglycan substrate in 51 of 57 cultures. In this figure the DG explant was placed so that it contacted laminin as well as the $1 \times$ and $2 \times$ concentrations of proteoglycan. As in explants placed directly on untreated proteoglycan substrate $(B, F)$, MFs extended onto laminin and $1 \times$ proteoglycan substrates but were inhibited by the $2 \times$ proteoglycan substrate, even after keratanase treatment. These data show that MF outgrowth is inhibited by CSPG substrates and that this inhibition is diminished by chondroitin $A B C$ lyase but not keratanase. Scale bar: $A, B, D, E, F, H, 200 \mu \mathrm{m} ; C, G, 80 \mu \mathrm{m}$. 


\section{HBSS}

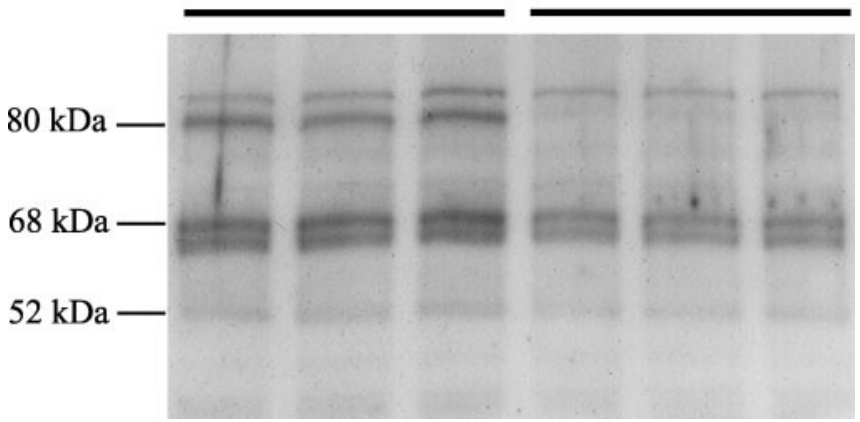

Figure 9. Western analysis demonstrated that keratanase reduces the phosphorylation of an $80 \mathrm{kDa}$ protein. An anti-phosphotyrosine antibody was used to examine whether keratanase altered the phosphorylation of specific proteins in dentate explants. In control explants treated with HBSS, a strong band was present at $\sim 80 \mathrm{kDa}$. This band was not detectable in homogenates from cultures treated with keratanases I+II (KIK2).

treatment of the explants. These explants are cut from whole hippocampal slices, and preserve the basic organotypic structure of the selected hippocampal region. Thus, explant studies provide additional information to help determine whether keratanase can directly alter the behavior of astrocytes that are migrating on a defined (laminin) substrate rather than trying to analyzing astrocyte behavior within intact slice cultures where it is difficult to distinguish direct from indirect effects. These data show that astrocytes in K1K2-treated dentate explants exhibited significantly greater migration on laminin after $48 \mathrm{hr}$ compared with HBSSand $\mathrm{ABC}$-treated explants (Fig. $10 A-D$ ). Astrocyte migration in ABC-treated dentate explants was not significantly different from migration in HBSS-treated explants (Fig. 10 D). Keratanase treatment also affected astrocyte migration in explants of the CA3 pyramidal cells (Fig. $10 \mathrm{~F}$ ). As with the dentate explants, astrocytes in the K1K2-treated CA3 explants showed significantly greater migration than HBSS- or ABC-treated explants. There was no significant difference in astrocyte migration between HBSS- and ABC-treated explants of CA3 pyramidal cells. The change in astrocyte migration on laminin suggests that keratanase directly altered astrocyte behavior by altering RPTP $\beta / \zeta$ activity because there are no other cells or exogenously applied matrix molecules to influence their behavior. The lack of effect by $\mathrm{ABC}$ also suggests that extracellular CSPGs such as neurocan do not regulate astrocyte behavior in this system. However, it is not possible to completely rule out that some indirect change in astrocyte behavior occurred within the explant before the astrocytes reached the laminin substrate. Such changes could, for example, alter the way astrocytes interact with or modify the laminin substrate. MF axon outgrowth was also analyzed in DG explants (Fig. $10 E$ ). MFs showed significantly greater outgrowth in explants treated with $\mathrm{K} 1 \mathrm{~K} 2$ than in explants treated with HBSS or ABC. MF outgrowth from ABC-treated explants was not significantly different from those treated with HBSS. Because axons comigrate with astrocytes on laminin, these observations are consistent with the possibility that changes in astrocyte behavior influence axon outgrowth. Axon outgrowth from CA3 pyramidal cells was not analyzed because these data were not directly comparable to any of our previous data. In summary, the observations in this study are consistent with the conclusion that keratanase alters MF outgrowth by changing the behavior and function of astrocytes.
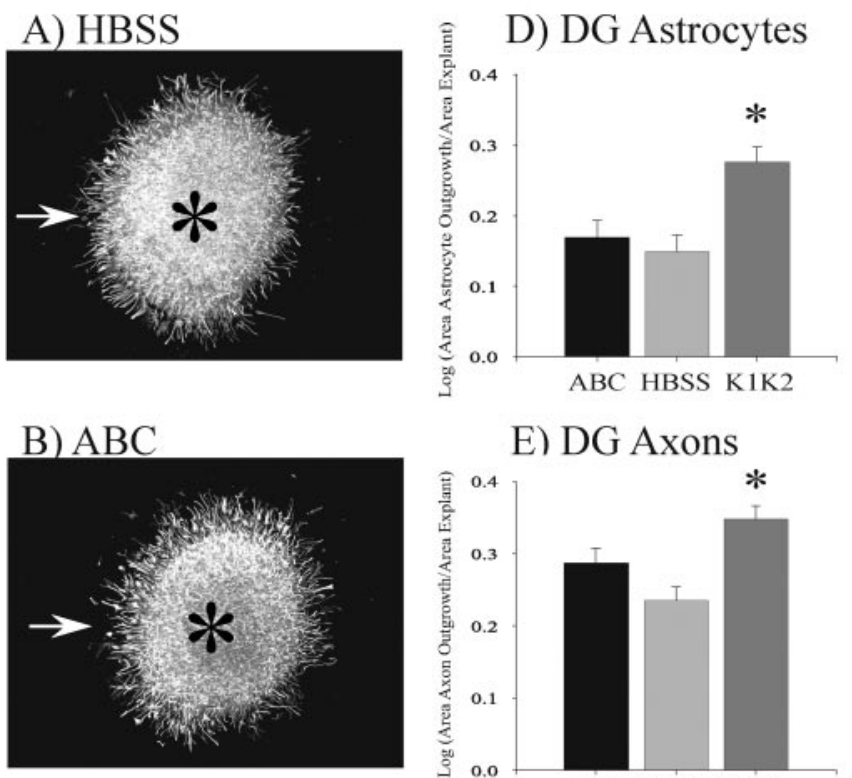

E) DG Axons

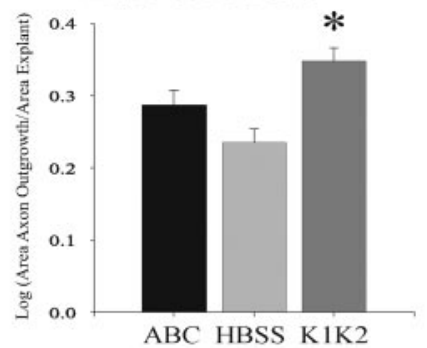

C) $\mathrm{K} 1 \mathrm{~K} 2$
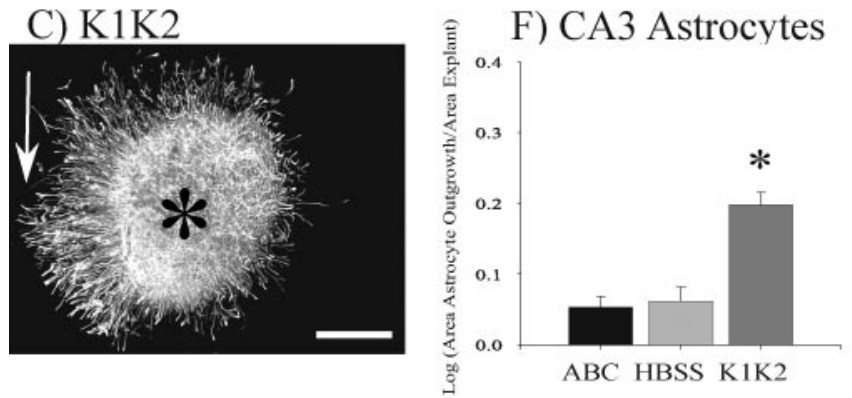

Figure 10. Keratanase enhanced astrocyte migration from dentate and CA3 explants. Figures 2 and 3 demonstrated that RPTP $\beta / \zeta$ is expressed by astrocytes and on astrocytic process located between the granule cell and CA3 pyramidal cell bodies. Dentate explants cultured on laminin were treated with keratanases I and II (K1K2), chondroitin ABC lyase (ABC), or HBSS to determine whether the enzyme treatment of the cell body area could alter astrocyte and MF outgrowth even when no CA3 target tissue was present. To measure astrocyte outgrowth, a circle was drawn around the outer border of the GFAP-positive astrocytic processes (arrows). A circle was then drawn around DAPI-stained explants to measure the area of the explant (data not shown). The explant can be observed as the densely stained areas in $A-C$, whose centers are marked by asterisks. The area of astrocyte outgrowth minus the area of the explant was then divided by the area of the explant to normalize the outgrowth data for different-sized explants. The log of this ratio was calculated to perform a linear transformation of the data for statistical analysis. The outgrowth of astrocytes in $\mathrm{K} 1 \mathrm{~K} 2$ treated dentate explants was statistically greater than the outgrowth observed in ABC- or HBSS-treated explants (D). K1K2 treatment produced a similar increase in astrocyte outgrowth in CA3 explants $(F)$. DG explants were also stained with $\beta$-tubulin to measure MF axon outgrowth $(E)$. MF outgrowth was significantly enhanced in explants treated with $\mathrm{K} 1 \mathrm{~K} 2$ compared with those treated with HBSS. MF outgrowth in ABCtreated explants was not significantly different from HBSS-treated explants. ANOVA statistical analysis was performed using SigmaStat 3.0 software. ${ }^{*} p<0.001$. Scale bar, $200 \mu \mathrm{m}$.

\section{Discussion}

We have examined the role of proteoglycans in directing MF outgrowth in the hippocampus. Proteoglycans are large glycoproteins that typically inhibit axon outgrowth, and this inhibition can be relieved by enzymatic removal of CS-GAG sugars using chondroitin ABC lyase (Snow et al., 1990, 1991; Brittis et al., 1992). Most proteoglycans are located in the extracellular matrix through which axon growth cones must travel, and they form inhibitory boundaries that funnel axon growth cones along the appropriate pathway to their synaptic target area. The two 
major proteoglycans in the brain are neurocan and phosphacan (Margolis et al., 1996). Using the 1D1 antibody, we observed that high levels of neurocan immunoreactivity bordered the SL in area CA3 of the hippocampus, making it a good candidate for directing MF outgrowth into this stratum. 3H1 and 3F8 monoclonal antibodies were used to analyze the distribution of extracellular KS-phosphacan and CS-phosphacan, respectively. The uniform distribution of 3F8 immunostaining suggested that CSphosphacan is not distributed in a manner that would help restrict MF outgrowth to the SL. The distribution of $3 \mathrm{H} 1$ immunoreactivity shows that KS-phosphacan is poorly expressed in all strata of CA3 proximal to the dentate, which suggests that extracellular KS-phosphacan is also a poor candidate for confining MF outgrowth to the SL. Thus, only neurocan appears to be properly positioned to direct MF growth cones into SL. However, this conclusion is based on the assumption that all proteoglycans are located in the extracellular matrix and act as repulsive barriers to growing axon growth cones. Consequently, interpreting the role of phosphacan in directing MF outgrowth is complicated by the fact that it is expressed not only as an extracellular proteoglycan but also as a transmembrane receptor protein tyrosine phosphatase $(\operatorname{RPTP} \beta / \zeta)$. RPTP $\beta / \zeta$ also contains KS-GAGs (Nishiwaki et al., 1998). Therefore, the presence of RPTP $\beta / \zeta$ around dentate and CA3 cell bodies raises the possibility that enzymatic removal of KS-GAGs from phosphacan/RPTP $\beta / \zeta$ could affect axon outgrowth by altering the phosphorylation state and function of astrocytes located near granule cell bodies.

The functional role of CSPGs and KSPGs in regulating MF outgrowth and regeneration has not been examined previously. Surprisingly, our data demonstrated that the pattern of MF outgrowth and regeneration was not altered by application of chondroitin ABC lyase to intact hippocampal slices. These data suggest that in spite of the fact that MFs are surrounded by neurocan, positive guidance molecules such as laminin appear to be sufficient under these conditions to properly guide MFs into SL (Grimpe et al., 2002). Dentate explant data shown in Figure 8, C and $G$, also suggest that MF outgrowth is not inhibited by CSPG core protein or stub sugar remaining after chondroitin $\mathrm{ABC}$ lyase digestion. In contrast, KSPGs appear to play a significant role in directing MF outgrowth. When either keratanase I or II was applied to hippocampal slices, transected MFs failed to regenerate their normal pattern. Specifically, MFs in keratanase-treated slices failed to coalesce in the SL when exiting the dentate. Rather than exiting the dentate as fasciculated fibers in the SL, MFs exited the dentate over a wider area along the border between the dentate and hippocampal area CA3. These fibers continued to grow in a disorganized manner to the end of area CA3. Thus, MFs grew into strata radiatum, pyramidale, lucidum, and oriens rather than being restricted exclusively in stratum lucidum.

The dramatic change in MF outgrowth produced by keratanases and the lack of response by chondroitin $\mathrm{ABC}$ lyase was puzzling and raised the possibility that MFs may exhibit unusual responses to KSPGs and CSPGs. We therefore examined the ability of MFs from dentate explants grown on laminin to cross onto a CSPG-KSPG substrate. Using this experimental model, we were able to interpret how MF growth cones responded to proteoglycans without influence from other guidance cues that may be present in the intact hippocampal slice. Thus, these data allow us to extrapolate how MF growth cones should respond to extracellular proteoglycans they encounter in area CA3 of intact hippocampal slice cultures. The mixture of proteoglycans used in this study contained neurocan, phosphacan, versican, and aggrecan isolated from embryonic chick brain. This mixture was cho- sen because it is similar to that found in neonatal rat hippocampus (Rauch et al., 1991; Oohira et al., 1994; Meyer-Puttlitz et al., 1995; Milev et al., 1998). The data in Figure 8 demonstrated that most MFs exhibited a typical response and turned abruptly to avoid the inhibitory CSPG-KSPG substrate. In addition, this inhibition of MF outgrowth was eliminated by digestion of the proteoglycan substrate with chondroitin $\mathrm{ABC}$ lyase. Thus, if proteoglycans residing in the extracellular matrix are the primary guidance molecules directing MFs into SL in intact hippocampus, chondroitin $\mathrm{ABC}$ lyase treatment of cultured hippocampal slices should have allowed MFs to regenerate abnormally into areas outside SL. These experiments also predict that keratanase treatment should have no effect on MF regeneration in cultured hippocampal slices.

Curiously, the results of these dentate explant experiments are just the opposite of those performed on intact cultured slices, because enzymatic treatment of the substrate demonstrated that CSPGs inhibit MF outgrowth but that KSPGs do not. These observations suggest that MF misrouting in keratanase-treated hippocampal slice cultures is not simply attributable to the loss of KS from extracellular KS-phosphacan in area CA3. Given the fact that phosphacan is primarily made by astrocytes, the alteration of MF regeneration in slice cultures would appear to involve a change in the way granule cells and MFs interact with glia. This change in the way neurons and glia interact could occur by two possible mechanisms. First, keratanase could alter astrocyte behavior indirectly. In this scenario, loss of KS from extracellular KS-phosphacan would be predicted to modify astrocyte behavior via changes in cell-cell or cell-matrix interactions. In contrast, mechanism 2 would involve a direct change in astrocyte behavior. Because RPTP $\beta / \zeta$ contains KS-GAGs (Nishiwaki et al., 1998), loss of KS from RPTP $\beta / \zeta$ would be predicted to alter the phosphatase activity and consequently the behavior of astrocytes. We have attempted to distinguish between these two possible mechanisms by observing the change in astrocyte migration on laminin after keratanase treatment of DG and CA3 explants (Fig. 10). We observed that keratanase enhanced astrocyte outgrowth on a laminin substrate. The change in astrocyte behavior on this defined substrate in the absence of extracellular phosphacan or other exogenous matrix molecules appears to support the direct activation of RPTP $\beta / \zeta$ (mechanism 2). This interpretation is consistent with the fact that keratanase treatment of dentate explants resulted in a decreased phosphorylation of an $80 \mathrm{kDa}$ protein. A mechanism involving the alteration of RPTP $\beta / \zeta$ activity by keratanase is also consistent with the fact that extracellular KS-phosphacan is not strongly expressed in area CA3 proximal to the dentate (Fig. 2D), and with the fact that the dentate is one of three brain areas that most strongly express RPTP $\beta / \zeta$ (Levy et al., 1993). However, we cannot entirely rule out mechanism 1 because it is possible that keratanase altered cell-cell or cell-matrix interactions within the explant. Thus, it is possible that astrocyte migration reflected indirect changes that occurred before the astrocytes exited the explant and started migrating on the defined laminin substrate.

How would changes in the activity of RPTP $\beta / \zeta$ on astrocytes mediate keratanase-induced changes in MF outgrowth? One possible mechanism is suggested by the work of the Schlessinger and Grumet labs, which provided strong evidence that RPTP $\beta / \zeta$ expressed on astrocytes interacts with contactin expressed on neuronal cells and that this interaction between the astrocytes and neurons can alter axon outgrowth (Peles et al., 1995, 1997; Sakurai et al., 1997). Thus, a similar interaction between astrocytes and granule cells might also alter the outgrowth of MFs in the 
hippocampus. Granule cells express mRNA for contactin (Shimazaki et al., 1998), as well as other members of the contactin family of glycosylphosphatidylinositol-linked adhesion proteins including BIG-1 (Yoshihara et al., 1994, 1995) and TAG1/F3 (Hosoya et al., 1995). We have also observed strong TAG-1/F3 staining of MFs in neonatal rats using the $3.1 \mathrm{C} 12$ monoclonal antibody (data not shown).

Our data cannot determine whether keratanase treatment directly alters the binding between $\mathrm{RPTP} \beta / \zeta$ and guidance molecules on neurons or whether reduced phosphorylation of the 80 $\mathrm{kDa}$ protein somehow leads to the observed changes in MF outgrowth. However, Margolis et al. (1996) have shown that keratanase treatment does not alter the binding of TAG-1, L1, or NCAM to extracellular phosphacan, whereas ABC lyase treatment reduced their binding by 70\% (Milev et al., 1996). The lack of change in phosphacan binding to guidance molecules provides additional evidence to support the conclusion that keratanaseinduced misrouting of MFs is not the result of changes in interactions between guidance molecules expressed by growth cones and extracellular proteoglycans in area CA3. In addition, these studies suggest that keratanase does not alter MF outgrowth by directly altering the binding of $\mathrm{RPTP} \beta / \zeta$ on astrocytes to cell adhesion molecules on neurons. Rather, they suggest that keratanase may change the downstream signaling resulting from binding of RPTP $\beta / \zeta$ to cell adhesion molecules.

Our data do not directly address how keratanase might alter phosphatase activity, however, it is possible that keratanase treatment could directly affect the ability of RPTP $\beta / \zeta$ to dimerize with itself after interacting with guidance molecules. This could have a dramatic effect on the neuron-glia responses because dimerization represents a major regulatory mechanism for phosphatase activity (den Hertog, 1999; Tertoolen et al., 2001). The decrease in phosphorylation of an $80 \mathrm{kDa}$ protein in Figure 9 suggests that keratanase treatment led to an increase in RPTP $\beta / \zeta$ enzymatic activity. The change in a single protein is consistent with, but does not prove that this protein was directly dephosphorylated by $\mathrm{RPTP} \beta / \zeta$. However, it is interesting to note that recombinant pleiotrophin, an inhibitor of RPTP $\beta / \zeta$ (Deuel et al., 2002), did not alter the outgrowth of either MFs or astrocytes from dentate explants (data not shown).

It is important to note that alteration of MF outgrowth in our complex slice culture system cannot be interpreted simply as an enhancement or inhibition of outgrowth. Rather, it appears that keratanase treatment leads to a disorganization of this pathway that normally grows in parallel bundles of fasciculated fibers that are restricted to the SL. Indeed, this organizational pattern forces MFs originating from dentate granule cells located near the open end of the dentate to send MFs backwards toward the center of the hilus before exiting the dentate through the SL. In contrast, regenerating MFs in keratanase-treated slices are not restricted to exiting the dentate in fasciculated bundles within the SL. These fibers, which lack the normal parallel pattern, take a rather tortuous path that winds through strata radiatum, lucidum, pyramidale, and oriens until they reach their normal termination point in area CA3A. These complex changes in the guidance and fasciculation of MFs are similar to the types of changes reported for type IIa RPTP mutations in Drosophila (Tian et al., 1991; Yang et al., 1991; Sun et al., 2000a). In addition, recent reports have shown that phosphatases also regulate axon outgrowth in vertebrate nervous systems (Sakurai et al., 1997; Mandell and Banker, 1998; Burden-Gulley and Brady-Kalnay, 1999; den Hertog, 1999; Sun et al., 2000b).

Although our data suggest that MF outgrowth can be affected
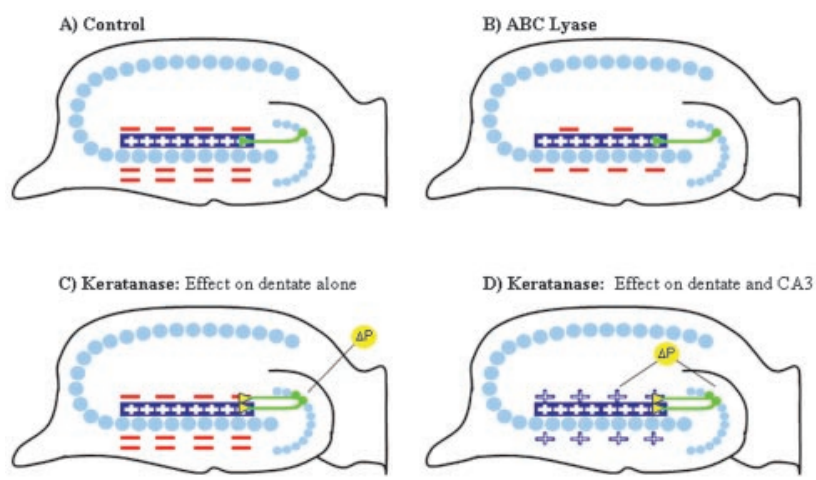

Figure 11. Models represent possible explanations of the data. $A$, In untreated hippocampus, MFs are hypothesized to follow attractive guidance cues in SL (+ signs). Inhibitory extracellular CSPGs also surround SL (red - signs). B, Treatment with chondroitin ABC lyase reduces the inhibitory extracellular CSPGs $(-)$, but MFs can still follow positive cues $(+)$ to regenerate their normal pattern. Thus, extracellular CSPGs surrounding SL do not appear to be the primary guidance molecules used by MFs. Furthermore, the data in Figure 8 , C and $G$, demonstrated that keratanase does not reduce inhibition of extracellular KSPGs, and Figure 8, D and $H$, demonstrated that extracellular KS-GAGs do not inhibit MF outgrowth. Thus, keratanase-induced changes in MF outgrowth appear to involve either the direct or indirect alteration of astrocyte function. Direct changes in RPTP $\beta / \zeta$ activity could alter the interaction of astrocytes and granule cells to produce a change in axon guidance receptors expressed on MF growth cones ( $C$ ) and/or alter the expression of guidance molecules by astrocytes in area $C A 3(D)$. Similar changes in the expression of guidance molecules or receptors also could be initiated by indirect mechanisms resulting from the loss of $\mathrm{KS}$ from extracellular phosphacan.

by interactions between astrocytes and dentate granule cells, CA3 explant data suggest that keratanase treatment can also alter the behavior of astrocytes in area CA3 (Fig. 10F). Thus, astrocytes surrounding the CA3 pyramidal cell bodies could influence the way these neurons respond to MF growth cones. Alternatively, astrocytes and/or neurons in area CA3 could express and excrete different guidance molecules or receptors after keratanase treatment. The alteration of protein expression could result from either direct or indirect changes in astrocyte behavior. Figure 11 provides several models that summarize how enzymatic treatment of cultured hippocampal slices produced the observed changes in MF outgrowth.

In summary, we have observed a dramatic change in the pattern of MF outgrowth and regeneration in hippocampal slice cultures treated with keratanase but not with chondroitin $\mathrm{ABC}$ lyase. In experiments using dentate explants, however, proteoglycans were inhibitory to MF outgrowth whether or not the proteoglycan substrate was treated with keratanase. In contrast, when dentate explants were treated with keratanase, astrocyte and MF outgrowth on laminin was altered. These data support the conclusion that the enzymatic removal of KS from either extracellular phosphacan or $\mathrm{RPTP} \beta / \zeta$ alters MF outgrowth by changing the function of astrocytes.

\section{References}

Altman J, Das GD (1965) Autoradiographic and histological evidence of postnatal hippocampal neurogenesis in rats. J Comp Neurol 124:319-336.

Alvarez-Royo P, Zola-Morgan S, Squire LR (1992) Impairment of longterm memory and sparing of short-term memory in monkeys with medial temporal lobe lesions: a response to Ringo. Behav Brain Res 52:1-5.

Asher RA, Morgenstern DA, Moon LD, Fawcett JW (2001) Chondroitin sulphate proteoglycans: inhibitory components of the glial scar. Prog Brain Res 132:611-619.

Brittis PA, Canning DR, Silver J (1992) Chondroitin sulfate as a regulator of neuronal patterning in the retina. Science 255:733-736. 
Burden-Gulley SM, Brady-Kalnay SM (1999) PTPmu regulates N-cadherindependent neurite outgrowth. J Cell Biol 144:1323-1336.

Canoll PD, Barnea G, Levy JB, Sap J, Ehrlich M, Silvennoinen O, Schlessinger J, Musacchio JM (1993) The expression of a novel receptor-type tyrosine phosphatase suggests a role in morphogenesis and plasticity of the nervous system. Brain Res Dev Brain Res 75:293-298.

Condic ML, Snow DM, Letourneau PC (1999) Embryonic neurons adapt to the inhibitory proteoglycan aggrecan by increasing integrin expression. J Neurosci 19:10036-10043.

den Hertog J (1999) Protein-tyrosine phosphatases in development. Mech Dev 85:3-14.

Deuel TF, Zhang N, Yeh HJ, Silos-Santiago I, Wang ZY (2002) Pleiotrophin: a cytokine with diverse functions and a novel signaling pathway. Arch Biochem Biophys 397:162-171.

Friedlander DR, Milev P, Karthikeyan L, Margolis RK, Margolis RU, Grumet M (1994) The neuronal chondroitin sulfate proteoglycan neurocan binds to the neural cell adhesion molecules Ng-CAM/L1/NILE and $\mathrm{N}$-CAM, and inhibits neuronal adhesion and neurite outgrowth. J Cell Biol 125:669-680.

Gaarskjaer FB (1985) The development of the dentate area and the hippocampal mossy fiber projection of the rat. J Comp Neurol 241:154-170.

Gaiarsa J-L, Heimrich B (1995) Restoration of mossy fiber projection in slice co-cultures of dislocated dentate gyrus and degranulated hippocampus. Dev Brain Res 86:250-258.

Granger R, Wiebe SP, Taketani M, Lynch G (1996) Distinct memory circuits composing the hippocampal region. Hippocampus 6:567-578.

Grimpe B, Dong S, Doller C, Temple K, Malouf AT, Silver J (2002) The critical role of basement membrane-independent laminin gamma 1 chain during axon regeneration in the CNS. J Neurosci 22:3144-3160.

Hosoya H, Shimazaki K, Kobayashi S, Takahashi H, Shirasawa T, Takenawa T, Watanabe K (1995) Developmental expression of the neural adhesion molecule F3 in the rat brain. Neurosci Lett 186:83-86.

Kunkel DD, Nguyen LB, Harrigan MR, Schwartzkroin PA, Malouf AT (1994) Somatostatin-containing neurons in rat organotypic hippocampal slice cultures: Light and electron microscopic immunocytochemistry. Hippocampus 4:157-166.

Kurazono S, Okamoto M, Sakiyama J, Mori S, Nakata Y, Fukuoka J, Amano S, Oohira A, Matsui H (2001) Expression of brain specific chondroitin sulfate proteoglycans, neurocan and phosphacan, in the developing and adult hippocampus of Ihara's epileptic rats. Brain Res 898:36-48.

Levy JB, Canoll PD, Silvennoinen O, Barnea G, Morse B, Honegger AM, Huang JT, Cannizzaro LA, Park SH, Druck T, (1993) The cloning of a receptor-type protein tyrosine phosphatase expressed in the central nervous system. J Biol Chem 268:10573-10581.

Mandell JW, Banker GA (1998) Selective blockade of axonogenesis in cultured hippocampal neurons by the tyrosine phosphatase inhibitor orthovanadate. J Neurobiol 35:17-28.

Margolis RK, Margolis RU (1993) Nervous tissue proteoglycans. Experientia 49:429-446.

Margolis RK, Rauch U, Maurel P, Margolis RU (1996) Neurocan and phosphacan: two major nervous tissue-specific chondroitin sulfate proteoglycans. Perspect Dev Neurobiol 3:273-290.

Mathern GW, Leite JP, Pretorius JK, Quinn B, Peacock WJ, Babb TL (1994) Children with severe epilepsy: evidence of hippocampal neuron losses and aberrant mossy fiber sprouting during postnatal granule cell migration and differentiation. Dev Brain Res 78:70-80.

Maurel P, Meyer-Puttlitz B, Flad M, Margolis RU, Margolis RK (1995) Nucleotide sequence and molecular variants of rat receptor-type protein tyrosine phosphatase-zeta/beta. DNA Seq 5:323-328.

Maurel P, Rauch U, Flad M, Margolis RK, Margolis RU (1994) Phosphacan, a chondroitin sulfate proteoglycan of brain that interacts with neurons and neural cell-adhesion molecules, is an extracellular variant of a receptor-type protein tyrosine phosphatase. Proc Natl Acad Sci USA 91:2512-2516.

Mello LE, Cavalheiro EA, Tan AM, Kupfer WR, Pretorius JK, Babb TL, Finch DM (1993) Circuit mechanisms of seizures in the pilocarpine model of chronic epilepsy: cell loss and mossy fiber sprouting. Epilepsia 34:985-995.

Meyer-Puttlitz B, Milev P, Junker E, Zimmer I, Margolis RU, Margolis RK (1995) Chondroitin sulfate and chondroitin/keratan sulfate proteoglycans of nervous tissue: developmental changes of neurocan and phosphacan. J Neurochem 65:2327-2337.
Milev P, Friedlander DR, Sakurai T, Karthikeyan L, Flad M, Margolis RK, Grumet M, Margolis RU (1994) Interactions of the chondroitin sulfate proteoglycan phosphacan, the extracellular domain of a receptor-type protein tyrosine phosphatase, with neurons, glia, and neural cell adhesion molecules. J Cell Biol 127:1703-1715.

Milev P, Maurel P, Haring M, Margolis RK, Margolis RU (1996) TAG-1/ axonin-1 is a high-affinity ligand of neurocan, phosphacan/proteintyrosine phosphatase-zeta/beta, and N-CAM. J Biol Chem 271:15716-15723.

Milev P, Maurel P, Chiba A, Mevissen M, Popp S, Yamaguchi Y, Margolis RK, Margolis RU (1998) Differential regulation of expression of hyaluronan-binding proteoglycans in developing brain: aggrecan, versican, neurocan, and brevican. Biochem Biophys Res Commun 247:207-212.

Nguyen LB, Ricciardi TN, Malouf AT (1996) Reinnervation of stratum lucidum by hippocampal mossy fibers is developmentally regulated. Dev Brain Res 95:184-193.

Nishiwaki T, Maeda N, Noda M (1998) Characterization and developmental regulation of proteoglycan-type protein tyrosine phosphatase zeta/ RPTPbeta isoforms. J Biochem (Tokyo) 123:458-467.

Okazaki MM, Evenson DA, Nadler JV (1995) Hippocampal mossy fiber sprouting and synapse formation after status epilepticus in rats: visualization after retrograde transport of biocytin. J Comp Neurol 352:515-534.

Oohira A, Katoh-Semba R, Watanabe E, Matsui F (1994) Brain development and multiple molecular species of proteoglycan. Neurosci Res 20:195-207.

Peles E, Nativ M, Campbell PL, Sakurai T, Martinez R, Lev S, Clary DO, Schilling J, Barnea G, Plowman GD, (1995) The carbonic anhydrase domain of receptor tyrosine phosphatase beta is a functional ligand for the axonal cell recognition molecule contactin. Cell 82:251-260.

Peles E, Nativ M, Lustig M, Grumet M, Schilling J, Martinez R, Plowman GD, Schlessinger J (1997) Identification of a novel contactin-associated transmembrane receptor with multiple domains implicated in proteinprotein interactions. EMBO J 16:978-988.

Rauch U, Gao P, Janetzko A, Flaccus A, Hilgenberg L, Tekotte H, Margolis RK, Margolis RU (1991) Isolation and characterization of developmentally regulated chondroitin sulfate and chondroitin/keratan sulfate proteoglycans of brain identified with monoclonal antibodies. J Biol Chem 266:14785-14801.

Sakurai T, Lustig M, Nativ M, Hemperly JJ, Schlessinger J, Peles E, Grumet M (1997) Induction of neurite outgrowth through contactin and Nr-CAM by extracellular regions of glial receptor tyrosine phosphatase beta. J Cell Biol 136:907-918.

Schlessinger AR, Cowan WM, Gottlieb DI (1993) An autoradiographic study of the time of origin and the pattern of granule cell migration in the dentate gyrus of the rat. J Comp Neurol 159:149-176.

Shimazaki K, Hosoya H, Takeda Y, Kobayashi S, Watanabe K (1998) Agerelated decline of F3/contactin in rat hippocampus. Neurosci Lett 245:117-120.

Snow DM, Letourneau PC (1992) Neurite outgrowth on a step gradient of chondroitin sulfate proteoglycan (CS-PG). J Neurobiol 23:322-336.

Snow DM, Lemmon V, Carrino DA, Caplan AI, Silver J (1990) Sulfated proteoglycans in astroglial barriers inhibit neurite outgrowth in vitro. Exp Neurol 109:111-130.

Snow DM, Watanabe M, Letourneau PC, Silver J (1991) A chondroitin sulfate proteoglycan may influence the direction of retinal ganglion cell outgrowth. Development 113:1473-1485.

Squire LR (1993) The hippocampus and spatial memory. Trends Neurosci 16:56-57.

Stoppini L, Buchs PA, Muller D (1991) A simple method for organotypic cultures of nervous tissue. J Neurosci Methods 37:173-182.

Sun Q, Bahri S, Schmid A, Chia W, Zinn K (2000a) Receptor tyrosine phosphatases regulate axon guidance across the midline of the Drosophila embryo. Development 127:801-812.

Sun QL, Wang J, Bookman RJ, Bixby JL (2000b) Growth cone steering by receptor tyrosine phosphatase delta defines a distinct class of guidance cue. Mol Cell Neurosci 16:686-695.

Sutula T, Cascino G, Cavazos J, Parada I, Ramirez L (1989) Mossy fiber synaptic reorganization in the epileptic human temporal lobe. Ann Neurol 26:321-330.

Tertoolen LG, Blanchetot C, Jiang G, Overvoorde J, Gadella Jr TW, Hunter T, 
Hertog J (2001) Dimerization of receptor protein-tyrosine phosphatase alpha in living cells. BMC Cell Biol 2:8.

Tian SS, Tsoulfas P, Zinn K (1991) Three receptor-linked protein-tyrosine phosphatases are selectively expressed on central nervous system axons in the Drosophila embryo. Cell 67:675-680.

Wight TN, Kinsella MG, Qwarnstrom EE (1992) The role of proteoglycans in cell adhesion, migration and proliferation. Curr Opin Cell Biol 4:793-801.

Wilson MT, Snow DM (2000) Chondroitin sulfate proteoglycan expression pattern in hippocampal development: potential regulation of axon tract formation. J Comp Neurol 424:532-546.

Wu YP, Siao CJ, Lu W, Sung TC, Frohman MA, Milev P, Bugge TH, Degen JL, Levine JM, Margolis RU, Tsirka SE (2000) The tissue plasminogen activator (tPA)/plasmin extracellular proteolytic system regulates seizureinduced hippocampal mossy fiber outgrowth through a proteoglycan substrate. J Cell Biol 148:1295-1304.
Yang XH, Seow KT, Bahri SM, Oon SH, Chia W (1991) Two Drosophila receptor-like tyrosine phosphatase genes are expressed in a subset of developing axons and pioneer neurons in the embryonic CNS. Cell 67:661-673.

Yoshihara Y, Kawasaki M, Tani A, Tamada A, Nagata S, Kagamiyama H, Mori K (1994) BIG-1: a new TAG-1/F3-related member of the immunoglobulin superfamily with neurite outgrowth-promoting activity. Neuron 13:415-426.

Yoshihara Y, Kawasaki M, Tamada A, Nagata S, Kagamiyama H, Mori K (1995) Overlapping and differential expression of BIG-2, BIG-1, TAG-1, and F3: four members of an axon-associated cell adhesion molecule subgroup of the immunoglobulin superfamily. J Neurobiol 28:51-69.

Zimmer J, Gähwiler BH (1987) Growth of hippocampal mossy fibers: A lesion and coculture study of organotypic slice cultures. J Comp Neurol 264:1-13. 